Published in final edited form as:

Neurotoxicology. 2014 May ; 42: 12-23. doi:10.1016/j.neuro.2014.02.009.

\title{
Quantitative Mapping of Trimethyltin Injury in the Rat Brain Using Magnetic Resonance Histology
}

\author{
G. Allan Johnson ${ }^{1,2}$, Evan Calabrese ${ }^{1,2}$, Peter B. Little ${ }^{3}$, Laurence Hedlund ${ }^{1}, \mathbf{Y i} \mathbf{Q i}^{1}$, and \\ Alexandra Badea ${ }^{1}$ \\ ${ }^{1}$ Center for In Vivo Microscopy, Department of Radiology, Duke University Medical Center, \\ Durham, NC \\ 2Biomedical Engineering, Duke University, Durham, NC \\ ${ }^{3}$ Neuropathology consultant, EPL Inc., Research Triangle Park, NC
}

\section{Abstract}

The growing exposure to chemicals in our environment and the increasing concern over their impact on health have elevated the need for new methods for surveying the detrimental effects of these compounds. Today's gold standard for assessing the effects of toxicants on the brain is based on hematoxylin and eosin (H\&E)-stained histology, sometimes accompanied by special stains or immunohistochemistry for neural processes and myelin. This approach is time-consuming and is usually limited to a fraction of the total brain volume. We demonstrate that magnetic resonance histology (MRH) can be used for quantitatively assessing the effects of central nervous system toxicants in rat models. We show that subtle and sparse changes to brain structure can be detected using magnetic resonance histology, and correspond to some of the locations in which lesions are found by traditional pathological examination. We report for the first time diffusion tensor imagebased detection of changes in white matter regions, including fimbria and corpus callosum, in the brains of rats exposed to $8 \mathrm{mg} / \mathrm{Kg}$ and $12 \mathrm{mg} / \mathrm{Kg}$ trimethyltin. Besides detecting brain-wide changes, magnetic resonance histology provides a quantitative assessment of dose-dependent effects. These effects can be found in different magnetic resonance contrast mechanisms, providing multivariate biomarkers for the same spatial location. In this study, deformation-based morphometry detected areas where previous studies have detected cell loss, while voxel-wise analyses of diffusion tensor parameters revealed microstructural changes due to such things as cellular swelling, apoptosis, and inflammation. Magnetic resonance histology brings a valuable addition to pathology with the ability to generate brain-wide quantitative parametric maps for markers of toxic insults in the rodent brain.

(C) 2014 Elsevier B.V. All rights reserved.

Corresponding Author: G. Allan Johnson, Center for In Vivo Microscopy, Department of Radiology, Box 3302 Duke University Medical Center, Durham, NC 27710 U.S.A. +1 919 684-7758, gjohnson@ duke.edu.

Publisher's Disclaimer: This is a PDF file of an unedited manuscript that has been accepted for publication. As a service to our customers we are providing this early version of the manuscript. The manuscript will undergo copyediting, typesetting, and review of the resulting proof before it is published in its final citable form. Please note that during the production process errors may be discovered which could affect the content, and all legal disclaimers that apply to the journal pertain. 


\section{Keywords}

animal models; rat; MRI; environmental toxins; trimethyltin

\section{Introduction}

The increasing presence of toxic compounds in the biosphere is elevating our need to understand their impact on the nervous system. Environmental factors have been implicated in neurologic diseases ranging from autism (Bushnell, 2013; LaSalle, 2013; Matsuzaki, et al. 2012), Parkinson's (Willis, et al. 2010), and Alzheimer's (Campdelacreu, 2012). The use of agricultural chemicals is growing nearly exponentially with wide-ranging potential impact on the nervous system (Costa, et al. 2008; Thany, et al. 2013). An ever-widening array of pharmaceuticals comes with an increasing potential for unwarranted neurologic side effects (Ekici, et al. 2011; Nevin, 2011). As the prevalence of these insults increases, the challenge to early identification of their impact on the nervous system grows ever more daunting.

The traditional approach for histopathologic evaluation of neurologic toxicology has recently been expanded from three slices to include seven carefully selected slices, providing a more robust method for assessing the impact of chemicals of concern (Bolon, et al. 2013; Rao, et al. 2011; Rao, et al. 2013). Even with this increase, the total volume of tissue studied is $<1 \%$ of the total brain volume. The simultaneous need to screen many more compounds and a desire to perform a more comprehensive coverage of the brain compels us to consider new approaches.

Over the last 30 years, magnetic resonance imaging (MRI) has revolutionized the clinical domain. The idea that one can view the brain noninvasively in three dimensions with exquisite soft tissue delineation seems almost like science fiction, but MRI is now in widespread daily use, with more than 8000 MRI systems in the United States (OECD, 2011). The closing sentence of Paul Lauterbur's seminal paper describing the invention of MRI clearly predicted its widespread adoption in the basic sciences: "Zeugmatographic (imaging) techniques should find many useful application in studies of the internal structures, states and composition of microscopic objects." (Lauterbur, 1973).

MRI can provide a particularly powerful approach to high-throughput scanning for neurotoxicologic evaluation, with wide use for clinical evaluation of neurotoxic insults at spatial resolution of 1-3 $\mathrm{mm}$. There are numerous live animal studies at spatial resolution $\sim 0.1-1 \mathrm{~mm}$. The suggestion that MRI could be applied to histopathology at resolution of $<0.1 \mathrm{~mm}$ was first made in 1993 (Johnson, et al. 1993). Magnetic resonance histology (MRH), i.e. the study of fixed tissues, is perfectly suited for neurotoxicology. MRH is nondestructive, is sensitive to many different types of injury, provides full three-dimensional coverage of the specimen, and is inherently digital, which present particularly appealing opportunities for high-throughput, automated analysis. Lerch et al. have published a particularly insightful analysis of the tradeoffs between live animal and ex vivo studies. When longitudinal information is not required, the lower cost and higher spatial resolution of MRH is preferred over in vivo studies (Lerch, et al. 2012). 
MRH was first employed to survey chemically-induced neuropathology in 2000 (Lester, et al. 2000). It has helped elucidate the pathogenesis of carbonyl sulfide (Morgan, et al. 2004; Sills, et al. 2004), and has become routine for structural neuroanatomical phenotyping (Badea, et al. 2007; Badea, et al. 2009; Ellegood, et al. 2012; Johnson, et al. 2007). But, the pathologic changes studied to date have for the most part been relatively large, focal lesions. For MRH to be useful for high-throughput screening, it must be able to detect more subtle pathology. The focus of this work is to assess the sensitivity of MRH for detecting such subtle, diffuse pathology.

As part of an ongoing effort to assess the potential for MRH, the International Life Sciences Institute (ILSI) / Health and Environmental Sciences Institute (HESI) Project Committee on Imaging for Translational Safety Assessment has been established to explore the utility of MRH in safety assessment (http://www.hesiglobal.org/i4a/pages/Index.cfm?pageID=3494). This group generated a list of potential compounds that would create diffuse lesions.

Trimethyltin hydroxide was chosen because it is well studied and produces a range of injury.

Trimethyltin (abbreviated TMT) is a selective toxicant, affecting several organs, including the central nervous system (CNS), where it has particular affinity for neurons containing the protein stannin (Toggas, et al. 1992). TMT is an organometal compound used for insect, bacteria, and fungus control, as well as for preserving wood, textiles, paints, and plastics. As polyvinyl chloride (PVC) products have become popular, reports of toxicity caused by organotin compounds used to stabilize such plastics (dimethyltin [DMT] and trimethyltin [TMT]) have emerged. Exposures to high doses of TMT, usually work-related, have been associated with behavioral and anatomical changes in the brain (Kreyberg, et al. 1992). The larger population may also be affected in cases of fires and contaminations. A recent study suggests that even in low doses, cumulative effects of alkyltins, such as DMT/TMT, can affect the population, e.g. through DMT/TMT leaching into drinking water from PVC pipes. We thus chose to study the CNS effects of TMT, known to be a toxicant for humans, as well as other mammals such as hamsters, gerbils, and marmosets (Brown, et al. 1984; Gozzo, et al. 1993), and the usual small animal mouse and rat models (Tang, et al. 2013; Toggas, et al. 1992).

While its mechanism is not fully understood, the spatio-temporal effects of TMT in the rat brain have been previously described (Dyer, et al. 1982). Early effects of TMT on the rat brain (1-3 days post-exposure) include neurodegeneration starting in the lateral septal nucleus, followed by the septo-hippocampal nucleus, septo-hypothalamic nucleus, anterior olfactory nucleus, bed nucleus of stria terminalis, endopiriform nucleus, parafascicular nucleus, superior colliculus, interstitial nucleus of the posterior commissure, inferior colliculus, pontine nuclei, raphe nuclei, spinal trigeminal nucleus, nucleus tractus solitatiris, vagal motor nucleus, the piriform cortex, enthorhinal cortex, and layers V and VI of the neocortex (Balaban, et al. 1988). Most studies focus on the striking effects, detected as early as 2-3 days post-exposure (Balaban, et al. 1988), on the hippocampal areas, including the granule cells (Bruccoleri, et al. 1998; Fiedorowicz, et al. 2008) and pyramidal cells in the CA areas and dentate gyrus, as well as subiculum (Brown, et al. 1979; Dyer, et al. 1982; Kim, et al. 2013); Mignini et al., 2012; (Shirakawa, et al. 2011). Changes in structures, including the piriform cortex and amygdala, have also been reported (Brown, et al. 1979). 
These anatomical changes in neuronal cell populations and glial cells (Brock and O'Callaghan 1987; McPherson, et al. 2013) are accompanied by behavioral changes collectively known as "TMT syndrome," including seizures, vocalizations, self-mutilation, whole-body tremors, and hyper-reactivity to touch (Brown, et al. 1979; Dyer, et al. 1982), as well as spatial memory impairment (Mignini, et al. 2012).

Because the injury is diffuse and widespread, TMT is a perfect candidate for a test of MRH and its ability to detect such injury. If, in fact, MRH can provide reasonable sensitivity and full coverage, it may allow expanded insight into TMT and its diverse effects. Conventional histological studies usually require some a priori knowledge of the areas to be sampled, or else it would be prohibitively time-consuming. Reproducing the same study in multiple animals, treated in different ways or doses, adds more to the burden. While lower in spatial resolution, MRH offers full three-dimensional (3D) coverage of the brain, and the ability to quantify the differential effects due to TMT toxicity. The focus of our study was to test the hypothesis that MRH could detect and quantitate the diffuse injury that is seen in TMT.

\section{Materials and methods}

All protocols were approved by the Duke University Institutional Animal Care and Use Committee. Dosing, tissue preparation, scanning, and image analysis were performed at the Duke Center for In Vivo Microscopy, and conventional histology was performed at the National Institute of Environmental Health Sciences (NIEHS) in the Research Triangle Park, North Carolina.

TMT hydroxide (Strem Chemical, Inc., Newburgport, MA) was dissolved in $0.9 \%$ saline. Twenty-four male Sprague Dawley rats ( 325 g) (Charles River, Wilmington, MA) were divided into three groups - saline control $(\mathrm{n}=8)$, low TMT dose $(8 \mathrm{mg} / \mathrm{kg})(\mathrm{n}=8)$, and high TMT dose $(12 \mathrm{mg} / \mathrm{kg})(\mathrm{n}=8)$. Animals received one IP injection of either saline or TMT. Animals in the saline and low-dose groups were sacrificed 6 days after dosing. Animals in the high-dose group were sacrificed 3 days after dosing, because their clinical symptoms were sufficient to cause humane concern. All the animals were perfusion-fixed using a protocol to actively stain the tissue to enhance the signal for MRH. Animals were anesthetized to a surgical plane and perfused through the left ventricle with outflow from a cut in the right atrium. The initial flush was a 1:10 solution of ProHance (Gadoteridol, Bracco Diagnostics, Monroe Township, NJ) and 0.9\% saline/0.1\% heparin. This was followed by fixation with 1:10 ProHance in 10\% phosphate buffered formalin. The heads were removed and placed in formalin for 48 hours and then transferred to a 1:200 ProHance/PBS solution to rehydrate the tissue. This active staining method was used to reduce the spin lattice relaxation time (T1) of the tissue to $\sim 100 \mathrm{~ms}$, providing significant signal enhancement (Johnson and Hedlund 2000; Johnson, et al. 2002).

MR imaging was performed on a 7T horizontal bore magnet interfaced to a General Electric (Epic 12.0) console (GE Medical Systems, Milwaukee, WI) modified for MR microscopy. Specimens were trimmed to fit into a $25-\mathrm{mm}$ diameter solenoid radio frequency (rf) coil. Two imaging sequences were used. A 3D gradient recalled echo (GRE) was acquired on a $512 \times 512 \times 1024$ array to provide a high-resolution anatomic scan (TR/TE=50/6ms). The 
isotropic resolution was 39 microns, i.e. voxels of $\sim 6$ picoliters $(\mathrm{pl})$. These voxels are $\sim 17,000$-times smaller than the voxels in a routine clinical MRI scan of the brain and 100times smaller than live animal studies. A second protocol was run to measure the diffusion properties of the tissue. This diffusion tensor imaging (DTI) protocol consisted of 7 different $3 \mathrm{D}(256 \times 256 \times 512)$ images acquired with a conventional $\mathrm{rf}$ refocused spin echo sequence (TR/TE=100/16.2 ms) using Stejskal Tanner diffusion encoding gradient with a pair of unipolar, half-sine diffusion gradient waveforms (width $[\delta]=3 \mathrm{~ms}$, separation $[\Delta]=8.5 \mathrm{~ms}$, gradient amplitude $=600 \mathrm{mT} / \mathrm{m}$ ). One baseline image with $\mathrm{b}=0$ (b0) and 6 high $\mathrm{b}$-value images $\left(b=1462 \mathrm{~s} / \mathrm{mm}^{2}\right)$ were acquired with diffusion sensitization along each of 6 noncollinear diffusion gradient vector directions $[1,1,0],[1,0,1],[0,1,1],[-1,1,0],[1,0,-1]$, and $[0,-1,1]$. Because this protocol required acquisition of 7 different images, the resolution was reduced by 2 along each axis to allow acquisition in a reasonable time period. Total acquisition time for all 8 3D images was approximately 16 hours. The isotropic spatial resolution for the diffusion tensor scans was $78 \mu \mathrm{m}$, i.e. voxel volumes of $\sim 48 \mathrm{pl}$. A complete description of the sequence can be found in reference (Johnson, et al. 2012).

Fig. 1 summarizes the image processing pipelines that were constructed to facilitate image reconstruction, post-processing of DTI parameters, image registration, and subsequent statistical evaluation of the volumetric images. Each specimen generates about $6.5 \mathrm{~GB}$ of raw and derived data. These processing pipelines are a key element of this study as they enable systematic, reproducible, automated processing of very large image sets. Scripts on the scanner automate the acquisition. Data are automatically transferred to a dedicated reconstruction engine that applies a Fermi filter, performs the three-dimensional Fourier Transform, scales the output, and produces the magnitude image for each 3D image. At this point, there are 8 3D images (1 gradient echo [GRE], 1 baseline image [b0], and 6 diffusion weighted images) for each specimen. The $73 \mathrm{D}$ images in the diffusion tensor set are advanced to the Diffusion Tensor pipeline, which registers each diffusion weighted image to the b0 scan, correcting for the linear portion of eddy currents and calculates the diffusion weighted image (DWI) and tensor in each voxel of the array. The diffusion tensor image (DTI) is used to calculate scalar images (for each specimen), including the apparent diffusion coefficient (ADC), axial diffusivity (AD), radial diffusivity (RD), fractional anisotropy (FA), and a color fractional anisotropy (clrFA).

The final (registration) pipeline has been described in detail elsewhere (Badea, et al. 2012). Registration is the process of bringing two images into the same space; the moving image is transformed or mapped into the same space as the reference image, via a transform-either a global linear (affine) transformation, or a combination of affine and local non-linear (diffeomorphic) transformation. Registration is therefore a pairwise operation. The method has been adapted from practices common in the clinical domain, with two significant differences. First, the method has been adapted to the specially optimized acquisition protocols we have developed for MRH of the rodent brain. Since the image contrast and spatial resolution in these protocols are radically different from clinical MRI, one must adapt the variables used in the registration/segmentation pipeline for this specific case. The second and obvious difference is that this pipeline must be driven by an atlas appropriate for the specific organ (brain), and of course, the species (rat). We have used our recently published 
atlas for the Wistar rat (Johnson, et al. 2012) as the reference. Since this reference atlas is at higher spatial resolution ( 25 and 50 microns) than that used in this work (39 and 78 microns), the atlas was scaled to the lower resolution. The skull was removed using an algorithm described previously (Badea, et al. 2003) Registration was performed on diffusion weighted images (DWI) with the Advanced Normalization Tools (ANTs) (Avants, et al. 2008) software package using an iterative approach.

Voxel-wise statistics on deformation fields and DTI parameters were performed after compensating for global changes in brain size via affine transforms. Remaining minor differences due to normal biological variation are expected to average out towards a null effect upon successful registrations. In contrast, pathology-induced changes will produce statistically significant alterations in the distributions of the tested parameters.

For voxel-wise statistics, we employed a minimum deformation template (MDT) strategy. MDT executes an iterative non-linear registration between each pair of image sets within an experimental group; in this case the control animals. In short, we performed the registration process for all pairs of images (DWI) in the control group, and then applied for each image i, the average transform of all possible $\mathrm{ij}$ transforms, where $\mathrm{j}$ varies from 1 to $\mathrm{N}$, and $\mathrm{j} \neq i$. Once the average deformation field for each specimen is calculated, it can be applied to each of the different contrasts (GRE, FA, RD, etc.) to transform them into the template space. At the end, all registered images were averaged, yielding an unbiased minimum deformation template. Thus for the control group, registration is performed on 8 data sets, resulting in a total of 28 pairwise registrations to generate the minimum deformation template. Similarly, the deformation fields for a given specimen in the remaining groups were calculated, to yield bidirectional transforms between that specimen and the template space (MDT for control group).

Parametric maps for diffusion tensor images and the log Jacobian of the deformation fields (used to characterize brain morphometric changes in the deformation-based morphometry approach, or DBM) were constructed at each voxel location in the atlas built for the control group, at the end of the registration process, and after Gaussian smoothing (4 voxels). A general linear model was fit for each voxel to test for the effect of TMT treatment. This resulting uncorrected statistic tests the null hypothesis (that TMT-treated rats have no differences relative to controls in the required deformation or FA value). As the number of voxels in MR images is large (e.g. 100,000), the standard hypothesis tests that control for type I errors at a level of $5 \%$ will result in a large number of false positives, on average 5000 for our 100,000 voxels, just by chance. The false discovery rate correction (FDR) provides an alternative to more conservative traditional multiple testing methods, and an objective way to select a $\mathrm{p}$ value controlling the proportion of false positive discoveries among all discoveries (Benjamini and Hochberg 1995; Benjamini and Yekutieli 2001; Genovese, et al. 2002; Storey 2002). FDR guarantees that on average, the proportion of false discoveries among all discoveries is upper bound by a rate q. Since at this stage we do not aim to rigorously test a hypothesis, but rather to explore the extent of potential injury, we used the false discovery rates (q-values) of 5\%, and 15\%. We used SurfStat (Chung, et al. 2010) to perform statistical testing for GRE images and DTI-derived images. 
At the conclusion of the MR imaging, 5 specimens ( 1 control, 1 from the $8 \mathrm{mg} / \mathrm{Kg}$ TMT dose-treated group, and 3 from $12 \mathrm{mg} / \mathrm{TMT}$-treated group) were returned to formalin and transferred to EPL Inc., Research Triangle Park, North Carolina for conventional histology and neuropathological examination. Specimens were removed from the skull, embedded in paraffin, sectioned at 5 microns, and stained for H\&E. Tissues were reviewed by a boardcertified pathologist (PBL).

Representative data sets from this study are available to the interested reader as a supplement to this article at http://www.civm.duhs.duke.edu/neurotox2013.

\section{Results}

Fig. 2 shows representative images from the GRE and three of the derived images from a control specimen, in horizontal sections. Since all the raw images are acquired without moving the specimen, all the images are registered to each other. By analogy to conventional histology, it is as if one had the same slice stained several different ways. The slices are not adjacent tissue sections. They are the same tissue section. In the GRE image (Fig. 2a), the higher resolution $(39 \mu \mathrm{m})$ provides good definition of fibers in the caudate/ putamen (arrow within $\mathrm{CPu}$ ). But, the contrast mechanism in the DW image (Fig. 2b) provides much higher contrast between the pyramidal cells and the granular layer of the dentate gyrus, and the rest of the hippocampus. The FA image (Fig. 2c) emphasizes white matter, as well as areas in which there is an ordered structure to the tissue. The tensor data provides information on the direction of order in the tissue, which is color-coded in the color FA image (Fig. 2d), so one can now differentiate CA1 from CA2 in the hippocampus.

In Fig. 3, the MRH data is resliced and magnified in a coronal plane through the olfactory bulb, allowing a closer inspection of the layers of the bulb. Again, the higher resolution in the GRE image permits delineation of thin layers, e.g. the ependymal layer (white arrow), and is sufficient to even demonstrate glomeruli (yellow arrow) in the outer layer. But the higher contrast in the diffusion-weighted and derived images provides a more robust signal for delineating the boundaries of the layers in the olfactory bulb. This is a critical strength for this method, as it allows automated segmentation of many different regions of the brain, while still intact in the cranium, and undistorted by shrinkage.

Fig. 4 demonstrates the results of image registration. Fig. 4a shows a 78-micron-thick sagittal section through a representative diffusion weighted image from the control group. Fig. $4 \mathrm{~b}$ shows the result of the pairwise registration of all 8 control specimens (inset is magnified in Fig. 5b). Fiber bundles apparent in the caudate/putamen of the single slice are blurred in the average slice. But the boundaries of the majority of the sub-regions are remarkably sharp.

Fig. 5 shows a magnified section of the average DWI image (from inset in Fig. 4b), along with histology and labels from Plate 169 from the Paxinos and Watson rat atlas (Paxinos and Watson, 2007). Fig. 5c has been manually edited to remove some of the labels to simplify comparison and focus on the hippocampus and region immediately below. Comparison of the histology (Fig. 5a) and average diffusion weighted atlas, obtained as the minimum 
deformation template for the control animals at the same location (Fig. 5b), demonstrates the single most important element of this work-the ability to make consistent volume measures of small regions in the brain and their changes. While the spatial resolution in the optical section is exquisite, one must keep in mind that this is a $40-\mu \mathrm{m}$ section (at lateral position of $1.90 \mathrm{~mm}$ ). The next section in the Paxinos/Watson atlas with the same stain (Plate 172) is at lateral position $2.62 \mathrm{~mm}$, i.e. 720 microns displaced. Though the in plane spatial resolution in the MR image is considerably less than that of the optical section, it is sufficient (even after averaging imposed by the registration) to delineate several layers of the hippocampus (e.g. the Oriens [Or], and Radiatum [Rad] on Fig. 5). Interestingly, the boundaries of several midbrain structures are clearer in the MR image than the histology image, for example, the posterior boundary of the pretectal nucleus (APTD and APTV) and the boundaries between several different layers of the superior colliculus (arrow on Fig. 5b).

Fig. 6 identifies the areas of the rat brain that show significant differences in volume and/or FA following TMT administration. The corrected p values $(\mathrm{Q})$ are shown as color overlays on the average DW images from the control group. Only areas that survived the false discovery rate correction at the level of 5\% (1 in 20 false discoveries allowed) are shown. The two columns on the left summarize areas of local volume changes in TMT-treated rats, identified by deformation-based morphometry (DBM). The two columns to the right show areas where local differences in water diffusion properties are significantly different, as reflected by differences in the fractional anisotropy parameters (FA). The coronal slices through the rat brain are shown from rostral (top of Fig. 6) to caudal (bottom of Fig. 6), showing selected levels in which there is morphologic difference between the control and low-dose atlases (column 1), or control and high-dose atlases (column 2). Areas that are significantly changed, presumably because of cell loss in TMT-treated rats, are present in the olfactory bulb in the low-dose treated animals, including the external plexiform, mitral, internal plexiform layer, and possibly the granule cell areas. Only the plexiform and possibly the glomerular cell layer appear to differ in the high-dose treated rats. The FA changes do not appear to be significant at this section level.

Fig. 6, row $\mathrm{b}$ through the caudate/putamen, containing areas of the piriform cortex show that local volume changes, and FA differences are significant in the caudate/putamen. In addition, morphometric changes are present in the piriform cortex, the primary somatosensory cortex, and the septohippocampal nucleus (in the high-dose treated group). The lateral septal nucleus shows unilateral changes in the FA values.

Fig. 6, row c indicates that there are significant volume changes localized to the hippocampal dentate gyrus (HcDG), the secondary somatosensory and the auditory cortex in the low-dose treated animals. Besides the HcDG, the affected areas extend to the pyramidal cell layers, and the corpus callosum and adjacent cortex (PtA - parietal association), in the high-dose treated group. A small focal area is present, although unilateral only in the amygdala. Small unilateral foci are the only indicators for FA changes in the fimbria of the hippocampus in the low-dose group, and the piriform cortex in the high-dose treated group. Morphologic changes are present in the auditory cortices. Interestingly the optic tract, medially located relative to the $\mathrm{CA} 3 \mathrm{Hc}$ area, appears to have different FA values in the lowdose TMT animals. 
Fig. 6, row d shows a coronal level through the substantia nigra. Here, both volume and FA changes are present in the HcDG in high-dose treated group, although the FA changes appear to cover smaller areas through the granule cell layers and the polymorphic layer in DG. Areas of volume loss are present in the HcDG and visual and auditory cortex in both treated groups.

Fig. 6, row e through the posterior commissure and the red nucleus indicates that tissue loss occurs at this level in the visual cortex. In the low-dose treated animals, the area through the tegmental decussation and the red nucleus is different in volume. In the high-dose treated groups DBM indicates changes in the interpeduncular nucleus area, possibly spanning over the trigemino-thalamic and medial lemniscus tracts, while FA changes clearly demarcate the HcDG as significantly different.

The last section of Fig. 6, row e is a section through the cerebellum and the fourth ventricle. Areas of significant volume change in TMT treated animals include the cochlear nucleus, the spinal trigeminal tract, the gigantocellular nucleus, and parts of the cerebellar white matter. The FA images indicate changes are also present in the cerebellar white matter and the spinal trigeminal nucleus (in the low-dose group only).

To facilitate comparison of the conventional histology (Fig. 7 and Table 1), we have expanded the previous findings in Fig. 8, presenting the magnitude of the effect sizes at the same locations as Fig. 6, though relaxing the false discovery rate (FDR) threshold to $15 \%$. Relaxing the FDR threshold was considered acceptable, as it provides means to generate new hypotheses, and increase the power to discover areas that are different (in volume or FA) in TMT-treated animals, throughout the whole brain. While the magnitude of the effect sizes varies as a continuum, the color scale we chose has been divided in only four discrete bins, assigned to effects considered: (a) low ( $<10 \%)$; (b) mild (10-30\%); (c) medium (30$50 \%$ ); or (d) large (above 50\%). We now see that most effect sizes are low, especially for the FA changes. Deformation-based morphometry, however, indicates that changes in the olfactory areas, the caudate/putamen, visual and auditory cortices, as well as dentate gyrus, cerebellar white matter, and brainstem nuclei represent mild effects. Medium effects are found in the primary somatosensory cortex (possibly the jaw area) and the spinal trigeminal tract area. Additional foci emerge, as we have lowered the FDR threshold in the medial lemniscus (row c), substantia nigra (row d), superior colliculus (row e), and cerebellar white matter, possibly encompassing the Purkinje cell layers as well. Changes in the striatum are particularly evident, as well as the hippocampal dentate gyrus, in both TMT-treated groups, and both deformation and FA markers.

Table 1 summarizes quantitative results of image-based markers for CNS pathology induced by TMT, and compares these with the gold standard provided by H\&E pathology. The lesions seen in the H\&E-stained sections are graded from $\mathrm{n}$ (not present) to $\mathrm{X} 1, \mathrm{X} 2, \mathrm{X} 3$ and $\mathrm{X} 4$, in increasing order of severity. The same notation is used to characterize MRH findings, based on the magnitude of effect sizes.

The nature of the neuropathological changes seen on H\&E stained sections extends from minimal to moderate neuronal central chromatolysis, as evident in the substantia nigra pars 
compacta, red nucleus, mesencephalic nucleus of cranial nerve $\mathrm{V}$ and medulla, to marked neuronal necrosis as seen in the dentate gyrus of the hippocampus. Not listed, but of interest, are focal cortical lesions in the parietal region of 12-mg-dosed animals, which consist of very subtle individual cell neuronal necrosis. It is apparent that the control has no comparable lesions in any part of the brain examined and the low-dose animal has minimal to mild lesions in only a few sites.

\section{Discussion}

The goal of this study was a test of MR histology in detecting subtle, diffuse neuropathologic injury induced by the test compound, trimethyltin. This is part of a more global goal of developing and validating MR histology as a tool for safety assessment. We have developed a standardized acquisition and reconstruction protocol, work flow, and processing pipelines that have been crucial in moving us from a demonstration to the more demanding execution of a complete study. Our standard protocol produces a diverse set of "proton stains," i.e. a range of contrasts designed to probe several different physical phenomena that detect tissue injury.

The results demonstrate four unique attributes of MR histology that lend themselves to the challenge of high-throughput screening. First, the protocol produces isotropic threedimensional (3D) image volumes in which there is virtually no shrinkage from fixation in alcohol that accompanies traditional H\&E sections. Since the tissue remains in the skull and there is no physical sectioning, there is very little morphologic distortion. The data can be examined along any arbitrary plane virtually eliminating challenges of finding homologous slices. Since the spatial resolution is isotropic, one can "slice" the tissue digitally allowing interactive choice of level and angle in any two-dimensional section.

The second crucial attribute is total, 3D coverage. The infrastructure and protocols support full coverage of the entire brain. At $39-\mu \mathrm{m}$ resolution, the array is large $(512 \times 512 \times 1024)$. But, the infrastructure we have established readily handles this large array (for multiple specimens in multiple groups) as single (3D) images. Reconstruction, post-processing, and archive are all automated. The state-of-the-art computer infrastructure facilitates both automated and user-driven interaction with the data. For example, one can simultaneously load as many as eight 3D images, and page through them interactively at matching levels.

The third attribute unique to this study is the diverse tissue contrast provided by this protocol. Soft tissue contrast has long been the forte of MRI. The earliest clinical applications of MRI, which relied on spin lattice (T1) and spin spin (T2)-weighted contrast, are now augmented by a wide range of imaging sequences sensitive to such things as flow, magnetic susceptibility, and diffusion. A particularly useful attribute for this study is the fact that one can obtain these different types of contrast on the same tissue, with perfect registration. As is clear in Fig. 2 and Fig. 3, the different scanning strategies highlight different anatomical structures. Our expectation is that these different contrasts will help highlight different types of changes. For example, in our previous work on carbonyl sulfide, the GRE image was particularly good at detecting blood because of the iron in the blood (Sills, et al. 2004). And in this study, changes in the diffusion properties of the tissue are 
clearly evident in Fig. 6. Our clinical colleagues have had 30 years to understand the relationships between disease and appearance in the MR images. We certainly have only scratched the surface.

Finally, the fourth attribute of MRH of interest is the inherent digital nature of this data. This, coupled to the other three attributes above, allows us to generate average templates for each group. Mapping between templates (control vs. low dose or control vs. high dose) provides statistical comparisons within and across groups that are simply not attainable using conventional optical sections.

The approach shown here has its own limitations. We have used the Wistar rat brain atlas to bring all brains in this study into the same coordinate space, and to indicate major anatomical regions of interest. While a regional analysis may be biased by the choice of the atlas and the strain it is based on, as well as age, and possibly gender, our study design is self sustained, in the sense that the group averages and local, voxel-wise changes are determined by the choice of our experimental groups alone. We compute deformations and DTI parametric changes among treated animals and controls that are strain, gender, and agematched. These changes can be referenced relative to an atlas. We have shown previously that a regional analysis based on automated segmentation can be performed for mice of different strains, using the C57BL6 mouse brain atlas (Badea et al., 2009; Wu, et al. 2012), but may require more aggressive local deformations for increased accuracy.

Critical examination of Fig. 6 shows several areas that are likely false-positives. The most ventral sections of the brain at the periphery in Fig. $6 \mathrm{c}$ and $6 \mathrm{~d}$ are suspicious because they are not symmetric. These false-positives are most probably due to susceptibility artifacts at the interface of the brain and skull and/or the errors in the skull-stripping algorithms. The pituitary is similarly suspect, since it lies in an area that is prone to both of these problems. Our expectation is that with time and experience the processing algorithms can be improved to reduce these problems.

Others and we have shown that MRH is sensitive to capturing regional volume and microstructural changes, using deformation-based morphometry and voxel-wise statistics on deformation fields and DTI parameters. For example, vasogenic and cellular edema alter the diffusion properties, reflected by the apparent diffusion coefficient (ADC) and FA changes cause by ethanol administration (Kong, et al. 2013). The authors found ADC increased in vasogenic edema, but decreased in cytotoxic edema, as well as decreased FA; and have correlated their findings with silver staining for imaging axons, their integrity and organization, as well as with aquaporin-4 expression (reflecting the evolution of edema). Traumatic brain injury (TBI)-induced axonal injury and edema result in altered DTI parameters (van de Looij, et al. 2012). Another TBI-associated pathology is gliosis, which is a major factor influencing DTI parameters (Budde, et al. 2011). Demyelination is thought to result primarily in radial diffusivity changes (Song, Yoshino J Fau - Le et al.), while axonal damage primarily in axial diffusivity changes (Sun, et al. 2006), as shown in a model of myelinating oligodendrocyte cell death (Mueggler, et al. 2012). 
Establishing which MR-based biomarkers correlate best with individual patho-histological changes is however, still an area of active research. For example, cell death would result in regional volume loss, but concomitant inflammation may induce swelling. Isolating the sources of changes in DTI parameters is also difficult, since they may act in opposite ways, such as microglia and astrocytes filling the space previously occupied by dead cells or myelin might provide diffusion barriers counteracting the possible effect by the loss of myelin membranes.

The increasing computational capabilities of current desktop computers, and the possibility of performing MRH-based population studies in a high-performance computing environment, pave the way for performing future studies in other models of toxic insults. We expect that future studies will be more time-efficient.

In summary, we have shown that although not as sensitive as conventional histological stains, magnetic resonance histology is able to capture distinct spatial patterns of changes in brains of rats treated with trimethyltin, based on different imaging contrasts obtained from diffusion tensor imaging. MRH studies can integrate the effects of neurotoxicant over a population, and help guide subsequent histological stains towards previously unsuspected areas.

\section{Conclusions}

In this study, we have focused our efforts on: 1) deformation-based morphometry to assess morphometric changes due to degeneration and/or gliosis, and 2) diffusion tensor imaging to assess microstructural changes through changes in the fractional anisotropy. We have focused on the FA parameter, since it is considered to be least affected by the fixation process, and thus most consistent/translational to in vivo studies (Sun, et al. 2003).

MRH results on early changes ( $<3-6$ days) in the rat brain exposed to TMT agree with H\&E-based evaluations, and the largest effects and best correlations between the two techniques were obtained for deformation-based morphometry, indicative of local volume changes. Effect sizes that survived the multiple comparison correction were in general either low or mild $(<30 \%)$, with medium effects in the corpus callosum and more lateral in the primary somatosensory cortex. The pontine areas appear to have medium effects, as well as other ventral brain areas, but these locations close to the brain boundary make these areas more susceptible to misregistration effects. The pathology as demonstrated on H\&E sections is sparse, in terms of number and location of apoptotic and necrotic cells (with the notable exception of the dentate gyrus of the hippocampus, where necrosis is widespread), but it is still detected at this "early" stage (3 days) by MRH.

While there is good general agreement, (though still qualitative) as indicated by Table 1 and Fig. 7, showing H\&E through areas identified as having significant differences in TMTtreated rats, the changes seem to be underestimated in MRH. The metrics for comparison of conventional and MR histology are entirely different. Conventional microscopy relies on trained assessment of subtle cell morphology seen at cellular resolution over a limited volume. MR results are based on integrated changes of morphology or changes in diffusion 
properties over the whole region. We believe the agreement is, however, suggestive of the value of MRH in evaluating CNS toxic lesions, while adding benefits specific to MR, which include straightforward quantification of lesion extent and effect sizes, and brain-wide coverage that enable discovery without a priori knowledge of the lesions.

MRI has become one of the most common tools for clinical evaluation of neurologic structure and function. There is a substantial literature describing structural and functional alteration in the human brain induced by exposure to varied neurotoxins (Fitsanakis, et al. 2006; Chuang, et al. 2007; Heaton, et al. 2007). There is some literature demonstrating the utility of MRI in neurotoxicology in small animal models (Bhagat, et al. 2005; Calas, et al. 2008; Gullapalli, et al. 2010; Chao, et al. 2011; Mullins, et al. 2013). But, acceptance of MRI as a primary screening tool for toxicology is limited. This is surprising given the wide clinical acceptance and ready translation between basic and clinical sciences. While we claim no definitive insight into the reasons, we speculate that the limited use is due to expense, standardization of protocols, and an existing knowledge base of how MR can serve the needs of pathologists. There are certainly challenges of scaling clinical MRI with 1-mm $\mathrm{m}^{3}$ voxels to the spatial resolution required for routine use by pathologists. The voxels in the GRE images shown here are nearly 17,000-times smaller than the highest resolution common in the clinical setting, and 100-times smaller than the resolution common in liveanimal studies. And what resolution is really required? What contrast mechanisms will be most sensitive in detecting what pathologies? What protocols should be standardized?

This work presents a starting point for standardized protocol and workflow, image pipelines to facilitate the workflow, and a test using a compound (trimethyltin) for which there is an extensive knowledge base. By doing so, we have made some small step toward demonstrating the utility of MRH, which brings a quantitative, multivariate approach for brain-wide studies of neurotoxic compounds. The questions listed above, and many others, must clearly be answered for MRH to become a routine tool for the pathologist and toxicologist. Most probably, the combined use of high resolution, specific histological staining, and brain wide multi-parametric statistical measures derived from MR will benefit neuropathology, These modalities will exert a push and pull effect, to advance the current knowledge on a wide range on neurotoxicant effects, and the interpretation of MR- and DTIbased statistical maps. But these same questions faced us when we wrestled the awkward MRIs of the early 1980s into our clinical environment (Johnson, et al. 1985). And through concerted research by an enormous number of scientists, engineers, and clinicians, questions about MRI have been answered, yielding one of the most effective tools in modern medicine. It seems likely that the same will be true as we work through the applications of MRH in pathology.

\section{Acknowledgements}

We are grateful to Dr. Robert Sills and Dr. Jean Harry at NIEHS for support in histopathology and useful discussions in understanding the effects of trimethyltin. We are particularly grateful to the members of the ISLI / HESI Project Committee on Imaging for Translational Safety Assessment http://www.hesiglobal.org/i4a/pages/ Index.cfm?pageID=3494 for advice in choosing the test compound and design of the protocol. We are also grateful to James Cook and Sally Zimney at the Duke Center for In Vivo Microscopy for technical assistance in image processing and help in preparing the manuscript. 
Funding

Dosing, tissue preparation, scanning, and image analysis were performed at the Duke Center for In Vivo Microscopy, an NIH/NIBIB national Biomedical Technology Resource Center (P41 EB015897).

\section{References}

Avants BB, Epstein CL, Grossman M, Gee JC. Symmetric diffeomorphic image registration with cross-correlation: evaluating automated labeling of elderly and neurodegenerative brain. Med Image Anal. 2008; 12(1):26-41. [PubMed: 17659998]

Badea A, Gewalt S, Avants BB, Cook JJ, Johnson GA. Quantitative mouse brain phenotyping based on single and multispectral MR protocols. Neuroimage. 2012; 63(3):1633-1645. http://dx.doi.org/ 10.1016/j.neuroimage.2012.07.021. [PubMed: 22836174]

Badea A, Johnson GA, Williams RW. Genetic dissection of the mouse brain using high-field magnetic resonance microscopy. Neuroimage. 2009; 45(4):1067-1079. http://dx.doi.org/10.1016/ j.neuroimage.2009.01.021. [PubMed: 19349225]

Badea A, Kostopoulos GK, Ioannides AA. Surface visualization of electromagnetic brain activity. J Neurosci Methods. 2003; 127(2):137-147. http://dx.doi.org/10.1016/S0165-0270(03)00100-6. [PubMed: 12906943]

Badea, A.; Williams, RW.; Johnson, GA. Proceedings of Society for Neuroscience. San Diego, CA: 2007 Nov. Magnetic resonance microscopy-based brain morphometry in BXD recombinant inbred mice; p. 3-7.

Balaban CD, O'Callaghan JP, Billingsley ML. Trimethyltin-induced neuronal damage in the rat brain: comparative studies using silver degeneration stains, immunocytochemistry and immunoassay for neuronotypic and gliotypic proteins. Neuroscience. 1988; 26(1):337-361. [PubMed: 2458546]

Benjamini Y, Hochberg Y. Controlling the false discovery rate - a practical and powerful approach to multiple testing. J Roy Statist Soc Ser B Series B (Methodological). 1995; 57(1):289-300.

Benjamini Y, D Yekutieli. The control of the false discovery rate in multiple testing under dependency. Ann Statis. 2001; 29(4):1165-1188. http://dx.doi.org/10.1214/aos/1013699998.

Bhagat YA, Obenaus A, Hamilton MG, Mikler J, Kendall EJ. Neuroprotection from soman-induced seizures in the rodent: evaluation with diffusion- and T2-weighted magnetic resonance imaging. Neurotoxicology. 2005; 26:1001-1003. [PubMed: 15982742]

Bolon B, Garman RH, Pardo ID, Jensen K, Sills RC, Roulois A, Radovsky A, Bradley A, AndrewsJones L, Butt M, Gumprecht L. STP position paper: Recommended practices for sampling and processing the nervous system (brain, spinal cord, nerve, and eye) during nonclinical general toxicity studies. Toxicol Pathol. 2013; 41(7):1028-1048. http:// doi.dx10.1177/0192623312474865. [PubMed: 23475559]

Brock TO, O'Callaghan JP. Quantitative changes in the synaptic vesicle proteins synapsin I and p38 and the astrocyte-specific protein glial fibrillary acidic protein are associated with chemicalinduced injury to the rat central nervous system. J Neurosci. 1987; 7(4):931-942. [PubMed: 3106588]

Brown AW, Aldridge WN, Street BW, Verschoyle RD. The behavioral and neuropathologic sequelae of intoxication by trimethyltin compounds in the rat. Am J Pathol. 1979; 97(1):59-82. [PubMed: 495696]

Brown AW, Verschoyle RD, Street BW, Aldridge WN, Grindley H. The neurotoxicity of trimethyltin chloride in hamsters, gerbils and marmosets. J Appl Toxicol. 1984; 4(1):12-21. [PubMed: 6425393]

Bruccoleri A, Brown H, Harry GJ. Cellular localization and temporal elevation of tumor necrosis factor-alpha, interleukin-1 alpha, and transforming growth factor-beta $1 \mathrm{mRNA}$ in hippocampal injury response induced by trimethyltin. J Neurochem. 1998; 71(4):1577-1587. [PubMed: 9751191]

Budde MD, Janes L, Gold E, Turtzo LC, Frank JA. The contribution of gliosis to diffusion tensor anisotropy and tractography following traumatic brain injury: validation in the rat using Fourier analysis of stained tissue sections. Brain. 2011 Aug; 134(Pt 8):2248-2260. http://dx.doi.org/ 10.1093/brain/awr161. [PubMed: 21764818] 
Bushnell PJ. Special issue: Environmental influences and emerging mechanisms in the etiology of autism. Neurotoxicol Teratol. 2013; 36:1-2. http://dx.doi.org/10.1016/j.ntt.2013.03.001. [PubMed: 23531343]

Calas AG, Richard O, Meme S, Beloeil JC, Doan BT, Gefflaust T, Meme W, Crusio WE, Pichon J, Montecot C. Chronic exposure to glufosinate-ammonium induces spatial memory impairments, hippocampal MRI modifications and glutamine synthetase activation in mice. Neurotoxicology. 2008; 29:740-747. http://doi.dx.org.10.1016/j.neuro.2008.04.020. [PubMed: 18562008]

Campdelacreu J. Parkinson disease and Alzheimer disease: environmental risk factors. Neurologia. 2012 Jun.:13. http://dx.doi.org/10.1016/j.nrl.2012.04.001.

Chao LL, Abadjian L, Hlavin J, Meyerhoff DJ, Weiner M. Effects of low-level sarin and cyclosarin exposure and Gulf War Illness on brain structure and function: A study at 4 T. Neurotoxicology. 2011; 32:814-812. http://dx.doi.org/10.1016/j.neuro.2011.06.006. [PubMed: 21741405]

Chuang WL, Huang CC, Chen CJ, Hsieh YC, Kuo HC, Shi TS. Carbon disulfide encephalopathy: cerebral microangiopathy. Neurotoxicology. 2007; 28:387-393. [PubMed: 17147956]

Chung MK, Worsley KJ, Nacewicz BM, Dalton KM, Davidson RJ. General multivariate linear modeling of surface shapes using SurfStat. Neuroimage. 2010; 53(2):491-505. http://dx.doi.org/ 10.1016/j.neuroimage.2010.06.032. [PubMed: 20620211]

Costa LG, Giordano G, Guizzetti M, Vitalone A. Neurotoxicity of pesticides: a brief review. Front Biosci. 2008. 13:1240-1249. [PubMed: 17981626]

Dyer RS, Walsh TJ, Wonderlin WF, Bercegeay M. The trimethyltin syndrome in rats. Neurobehav Toxicol Teratol. 1982; 4(2):127-133. [PubMed: 7201084]

Ekici B, Aydin K, Cantez S, Ergul Y, Tatli B. Etanercept-induced encephalopathy in a 7-year-old child. Pediatr Neurol. 2011; 45(4):271-273. http://dx.doi.org/10.1016/j.pediatrneurol.2011.06.013. [PubMed: 21907894]

Ellegood J, Henkelman RM, Lerch JP. Neuroanatomical assessment of the Integrin beta3 mouse model related to autism and the serotonin system using high resolution MRI. Front Psychiatry. 2012; 3:37. http://dx.doi.org/10.3389/fpsyt.2012.00037. [PubMed: 22557981]

Fiedorowicz A, Figiel I, Zaremba M, Dzwonek K, Schliebs R, Oderfeld-Nowak B. Trimethyltinevoked apoptosis of murine hippocampal granule neurons is accompanied by the expression of interleukin-1beta and interleukin-1 receptor antagonist in cells of ameboid phenotype, the majority of which are NG2-positive. Brain Res Bull. 2008; 77(1):19-26. [PubMed: 18639741]

Fitsanakis VA, Zhang N, Avison MJ, Gore JC, Aschner JL, Aschner M. The use of magnetic resonance imaging (MRI) in the study of manganese neurotoxicity. Neurotoxicology. 2006; 27:798-806. [PubMed: 16620989]

Genovese CR, Lazar NA, Nichols T. Thresholding of statistical maps in functional neuroimaging using the false discovery rate. Neuroimage. 2002; 15(4):870-878. [PubMed: 11906227]

Gozzo S, Perretta G, Monaco V, Andreozzi U, Rossiello E. The neuropathology of trimethyltin in the marmoset (Callithrix jacchus) hippocampal formation. Ecotoxicol Environ Safety. 1993; 26(3): 293-301. [PubMed: 7507820]

Gullapalli RP, Aracava Y, Zhuo J, Neto EH, Wang J, Makris G, Merchenthaler I, Pereira EFR, Albuquerque EX. Magnetic resonance imaging reveals that galantamine prevents structural brain damage induced by an acute exposure of guinea pigs to soman. Neurotoxicology. 2010; 31:67-76. http://dx.doi.org/10.1016/j.neuro.2009.09.004. [PubMed: 19782102]

Heaton KJ, Palumbo CL, Proctor SP, Killiany RJ, Yurgelun-Todd DA, White RF. Quantitative magnetic resonance brain imaging in US army veterans of the 1991 Gulf War potentially exposed to sarin and cyclosarin. Neurotoxicology. 2007; 28(4):761-769. [PubMed: 17485118]

Johnson GA, Herfkens RJ, Brown MA. Tissue relaxation time: in vivo field dependence. Radiology. 1985; 156:805-810. [PubMed: 2991980]

Johnson GA, Benveniste H, Black RD, Hedlund LW, Maronpot RR, Smith BR. Histology by magnetic resonance microscopy. Magn Reson Q. 1993; 9(1):1-30. [PubMed: 8512830]

Johnson, GA.; Hedlund, LW., inventors. Duke University, assignee. Three dimensional morphology using magnetic resonance U.S.A. patent U.S. 09/824,562. 2000. 
Johnson GA, Cofer GP, Fubara B, Gewalt SL, Hedlund LW, Maronpot RR. Magnetic resonance histology for structural phenotyping. J Magn Reson Imaging. 2002; 16(4):423-429. [PubMed: 12353257]

Johnson GA, Ali-Sharief A, Badea A, Brandenburg J, Cofer G, Fubara B, Gewalt S, Hedlund LW, Upchurch L. High-throughput morphologic phenotyping of the mouse brain with magnetic resonance histology. Neuroimage. 2007; 37(1):82-89. [PubMed: 17574443]

Johnson GA, Calabrese E, Badea A, Paxinos G, Watson C. A multidimensional magnetic resonance histology atlas of the Wistar rat brain. Neuroimage. 2012; 62(3):1848-1856. http://dx.doi.org/ 10.1016/j.neuroimage.2012.05.041. [PubMed: 22634863]

Kim J, Yang M, Kim SH, Kim JC, Wang H, Shin T, Moon C. Possible role of the glycogen synthase kinase-3 signaling pathway in trimethyltin-induced hippocampal neurodegeneration in mice. PLoS One. 2013; 8(8):70356. http://dx.doi.org/10.1371/journal.pone.0070356.

Kong L, Lian G, Zheng W, Liu H, Zhang H, Chen R. Effect of alcohol on diffuse axonal injury in rat brainstem: diffusion tensor imaging and aquaporin-4 expression study. Biomed Res Int. 2013:798261. http://dx.doi.org/10.1155/2013/798261 2013. [PubMed: 24282821]

Kreyberg S, Torvik A, Bjorneboe A, Wiik-Larsen W, Jacobsen D. Trimethyltin poisoning: report of a case with postmortem examination. Clin Neuropathol. 1992; 11(5):256-259. [PubMed: 1424320]

LaSalle JM. Epigenomic strategies at the interface of genetic and environmental risk factors for autism. J Hum Genet. 2013; 58(7):396-401. http://dx.doi.org/10.1038/jhg.2013.49. [PubMed: 23677056]

Lauterbur PC. Image formation by induced local interactions - examples employing nuclear magnetic resonance. Nature. 1973; 242:190-191.

Lerch JP, Gazdzinski L, Germann J, Sled JG, Henkelman RM, Nieman BJ. Wanted dead or alive? The tradeoff between in-vivo versus ex-vivo MR brain imaging in the mouse. Front Neuroinform. 2012; 6:6. http://dx.doi.org/10.3389/fninf.2012.00006. [PubMed: 22470335]

Lester DS, Pine PS, Delnomdedieu M, Johannessen JN, Johnson GA. Virtual neuropathology: threedimensional visualization of lesion due to toxic insult. Toxicol Path. 2000; 28(1):100-104. [PubMed: 10668995]

Matsuzaki H, Iwata K, Manabe T, Mori N. Triggers for autism: genetic and environmental factors. J Cent Nerv Syst Dis. 2012; 4:27-36. http://dx.doi.org/10.4137/JCNSD.S9058. [PubMed: 23650465]

McPherson CA, Merrick BA, Harry GJ. In vivo molecular markers for pro-inflammatory Cytokine M1 stage and resident microglia in trimethyltin-induced hippocampal injury. Neurotox Res. 2014 Jan; 25(1):45-56. http://dx.doi.org/10.1007/s12640-013-9422-3. [PubMed: 24002884]

Mignini F, Nasuti C, Artico M, Giovannetti F, Fabrizi C, Fumagalli L, Iannetti G, Pompili E. Effects of trimethyltin on hippocampal dopaminergic markers and cognitive behaviour. Int $\mathbf{J}$ Immunopathol Pharmacol. 2012; 25(4):1107-1119. [PubMed: 23298501]

Morgan DL, Little PB, Herr DW, Moser VC, Collins B, Herbert R, Johnson GA, Maronpot RR, Harry GJ, Sills RC. Neurotoxicity of carbonyl sulfide in F344 rats following inhalation exposure for up to 12 weeks. Toxicol Appl Pharmacol. 2004; 200(2):131-145. [PubMed: 15476866]

Mueggler T, Pohl H, Baltes C, Riethmacher D, Suter U, Rudin M. MRI signature in a novel mouse model of genetically induced adult oligodendrocyte cell death. Neuroimage. 2012 Jan 16; 59(2): 1028-1036. http://dx.doi.org/10.1016/j.neuroimage.2011.09.001. [PubMed: 21945466]

Mullins RJ, Xu S, Pereira EF, Mamczarz J, Albuquerque EX, Gullapalli RP. Delayed hippocampal effects from a single exposure of prepubertal guinea pigs to sub-lethal dose of chlorpyrifos: a magnetic resonance imaging and spectroscopy study. Neurotoxicology. 2013; 36:42-48. http:// dx.doi.org/10.1016/j.neuro.2013.02.002. [PubMed: 23411083]

Nevin RL. Mefloquine neurotoxicity and gap junction blockade: critical insights in drug repositioning. Neurotoxicology. 2011; 32(6):986-987. author reply 987.http://dx.doi.org/10.1016/j.neuro. 2011.05.003. [PubMed: 21605593]

ECD 2011; - Medical technologies, in. Health at a Glance 2011: OECD Indicators, OECD Publishing. http://dx.doi.org/10.1787/health_glance-2011-30-en.

Paxinos, G.; Watson, C. The Rat Brain in Stereotaxic Coordinates. New York: Academic Press; 2007. 
Rao DB, Little PB, Malarkey DE, Herbert RA, Sills RC. Histopathological evaluation of the nervous system in national toxicology program rodent studies: a modified approach. Toxicol Path. 2011; 39:463-470. http://dx.doi.org/10.1177/0192623311401044. [PubMed: 21430177]

Rao DB, Little PB, Sills RC. Subsite awareness in neuropathology evaluation of National Toxicology Program (NTP) studies: A review of select neuroanatomical structures with their functional significance in rodents. Toxicol Pathol. 2013 Oct.:16. [Epub ahead of print] http://dx.doi.org/ $10.1177 / 0192623313501893$.

Shirakawa T, Nakano K, Hachiya N, Kato N, Kaneko K. The involvement of P2X(1) receptor in pyramidal cell degeneration in the rat hippocampus after trimethyltin administration. Neurosci Res. 2011; 71(4):396-404. http://dx.doi.org/10.1016/j.neures.2011.08.005. [PubMed: 21871932]

Sills RC, Morgan DL, Herr DW, Little PB, George NM, Ton TV, Love NE, Maronpot RR, Johnson GA. Contribution of magnetic resonance microscopy in the 12-week neurotoxicity evaluation of carbonyl sulfide in Fischer 344 rats. Toxicol Path. 2004; 32:501-510. [PubMed: 15603534]

Song SK, Yoshino J, Le TQ, Lin SJ, Sun SW, Cross AH, Armstrong RC. Demyelination increases radial diffusivity in corpus callosum of mouse brain. Neuroimage. 2005; 26(1):132-140. [PubMed: 15862213]

Storey JD. A direct approach to false discovery rates. J Royal Statis Soc Series B (Statistical Methodology). 2002; 64:479-498.

Sun SW, Liang HF, Trinkaus K, Cross AH, Armstrong RC, Song SK. Noninvasive detection of cuprizone induced axonal damage and demyelination in the mouse corpus callosum. Magn Reson Med. 2006; 55(2):302-308. [PubMed: 16408263]

Sun SW, Neil JJ, Song SK. Relative indices of water diffusion anisotropy are equivalent in live and formalin-fixed mouse brains. Magn Reson Med. 2003; 50(4):743-748. [PubMed: 14523960]

Tang X, Wu X, Dubois AM, Sui G, Wu B, Lai G, Gong Z, Gao H, Liu S, Zhong Z, Lin Z, Olson J, Ren X. Toxicity of trimethyltin and dimethyltin in rats and mice. Bull Environ Contam Toxicol. 2013; 90(5):626-633. http://dx.doi.org/10.1007/s00128-013-0975-x. [PubMed: 23478947]

Thany SH, Reynier P, Lenaers G. Neurotoxicity of pesticides: its relationship with neurodegenerative diseases. Med Sci (Paris). 2013; 29(3):273-278. http://dx.doi.org/10.1051/medsci/2013293013. [PubMed: 23544381]

Toggas SM, Krady JK, Billingsley ML. Molecular neurotoxicology of trimethyltin: identification of stannin, a novel protein expressed in trimethyltin-sensitive cells. Mol Pharmacol. 1992; 42(1):4456. [PubMed: 1635553]

van de Looij Y, Mauconduit F, Beaumont M, Valable S, Farion R, Francony G, Payen JF, Lahrech H. Diffusion tensor imaging of diffuse axonal injury in a rat brain trauma model. NMR Biomed. 2012; 25(1):93-103. http://dx.doi.org/10.1002/nbm.1721. [PubMed: 21618304]

Willis AW, Sterling C, Racette BA. Conjugal Parkinsonism and Parkinson disease: a case series with environmental risk factor analysis. Parkinsonism Relat Disord. 2010; 16(3):163-166. http:// dx.doi.org/10.1016/j.parkreldis.2009.09.002. [PubMed: 19818671]

Wu T, Bae MH, Zhang M, Pan R, Badea A. A prior feature SVM-MRF based method for mouse brain segmentation. Neuroimage. 2012; 59(3):2298-2306. http://dx.doi.org/10.1016/j.neuroimage. 2011.09.053. [PubMed: 21988893] 


\section{Highlights}

- Three groups of SD rats ( $\mathrm{N}=8$ /group): control, $8 \mathrm{mg} / \mathrm{Kg}$ and $12 \mathrm{mg} / \mathrm{Kg}$ trimethyltin

- MR histology with GRE and diffusion tensor imaging at 39- and 78-micron resolution

- Automated registration and voxel-based analysis for statistical parametric maps

- Quantitative assessment of regional changes in volume and fractional 


\section{Reconstruction}

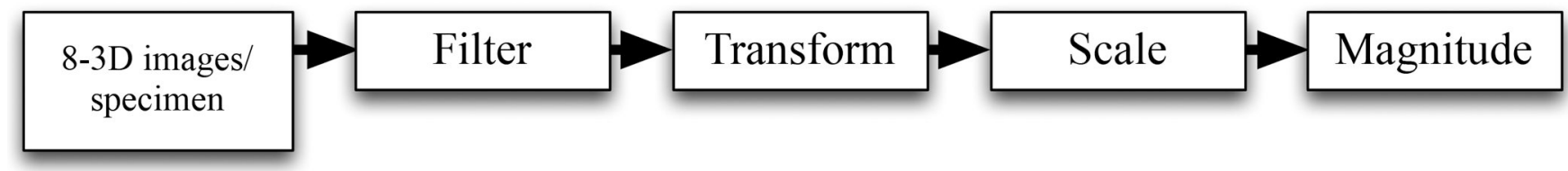

\section{Diffusion Tensor}

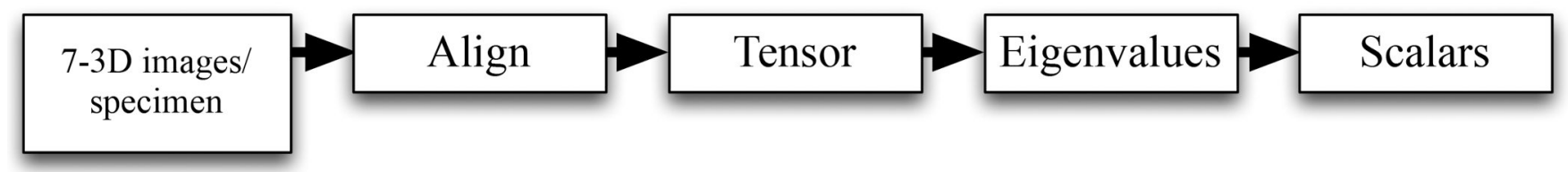

\section{Registration}

8 specimens/group

7 images/specimen

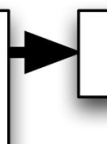

\section{Skull strip}

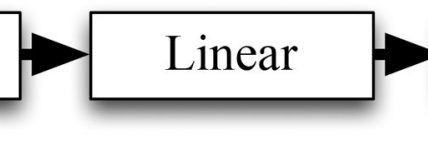

\section{Affine}

Fig. 1.

Three image-processing pipelines were constructed to facilitate processing of the multiple three-dimensional arrays and to ensure that the data were all processed in identical fashion. Software modules on multiple computers are connected via Perl scripts to automate the steps within and between the pipelines, including: image reconstruction, diffusion tensor estimation, and image registration. Image registration consists of both affine and diffeomorphic mapping (diff morph). 

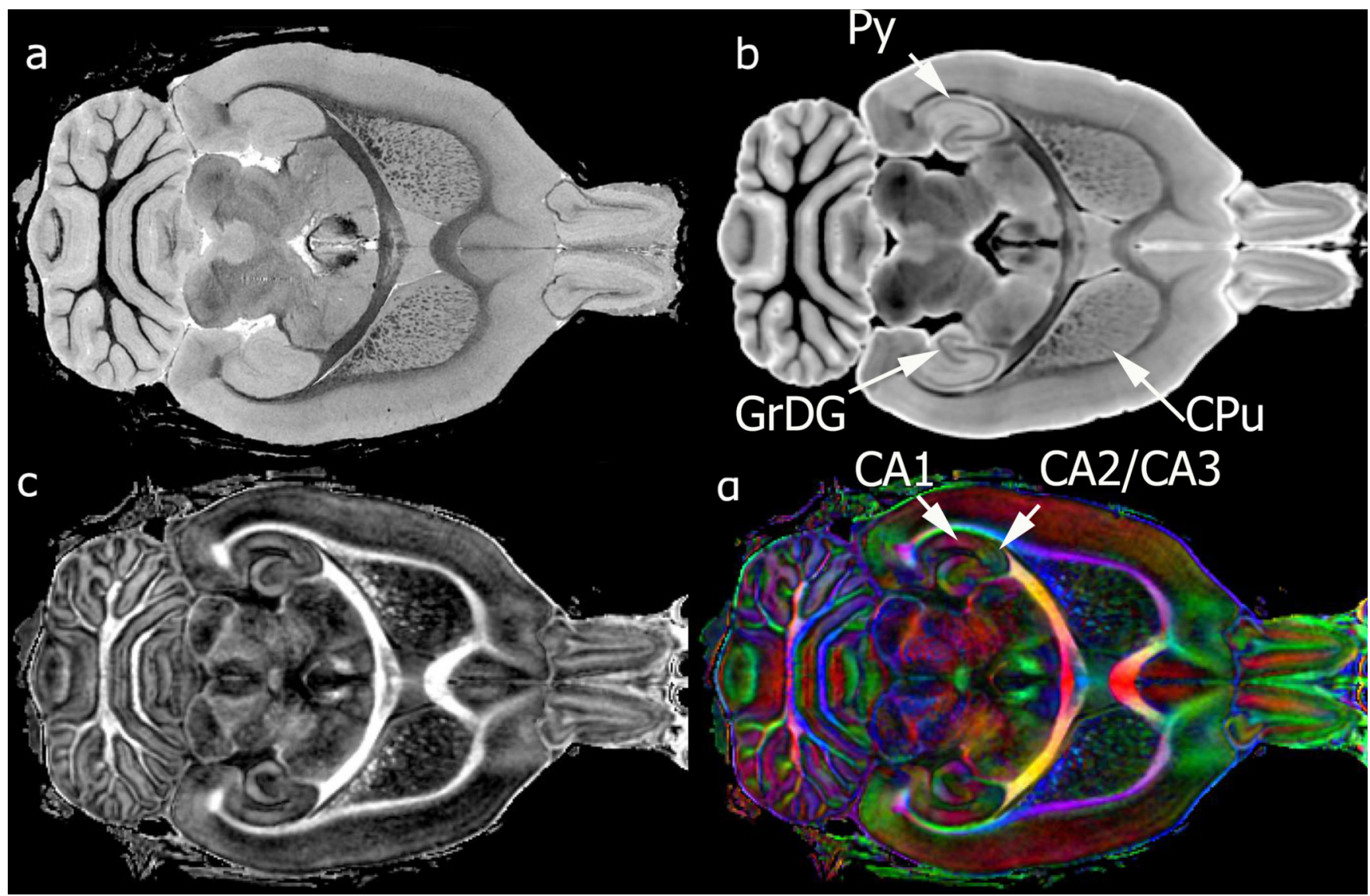

Fig. 2.

Representative images are shown from a control animal in a dorsal plane: (a) GRE image; (b) diffusion weighted image (DWI); (c) fractional anisotropy (FA) image; (d) color fractional anisotropy (clrFA). Py (pyramidal cell layer); CA1, CA2, CA3 (cornu ammonis fields of the hippocampus); $\mathrm{GrDG}$ (granular layers of the dentate gyrus); $\mathrm{CPu}$ (caudate putamen [fibers] - see arrow). 


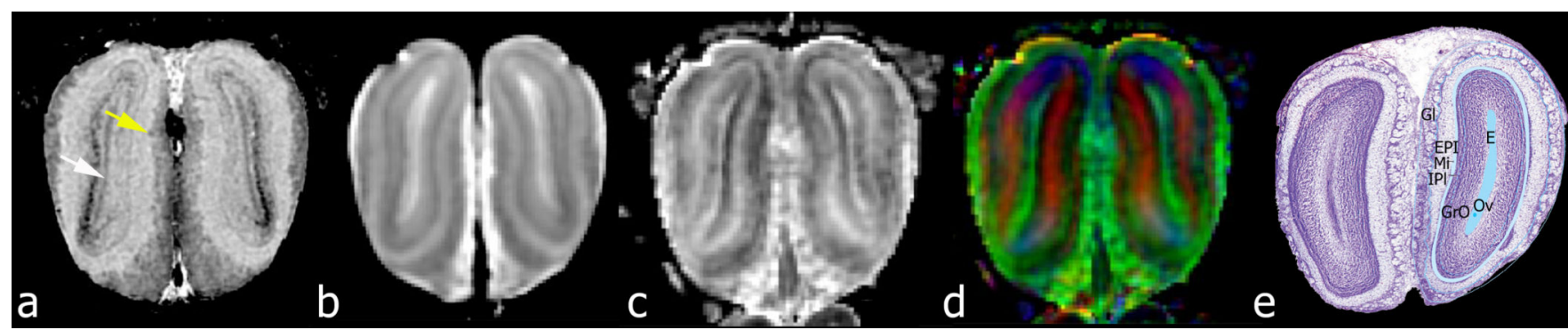

Fig. 3.

Coronal slices from the same data as in Fig. 2 demonstrate the neuroanatomy in the olfactory bulb in (a) GRE image, where cell layers can be seen, e.g. the ependymal layer (white arrow), as well as glomeruli (yellow arrow) in the outer layer; (b) diffusion weighted image (DWI); (c) fractional anisotropy (FA) image; (d) color fractional anisotropy (clrFA); (e) labeled cell layers in top of H\&E stain from similar slice through the rat brain atlas (adapted from and reprinted with permission from Elsevier Academic Press, San Diego, Plate 1 in: George Paxinos and Charles Watson, The Rat Brain in Stereotaxic Coordinates $6^{\text {th }} e d$., ISBN-13 978-0125476126, 2007). E (ependymal layer); Ov (olfactory ventricle); G1 (glomerular layer); EPl (external plexiform layer); IPl (internal plexiform layer); MI (mitral cells layer); $\mathrm{GrO}$ (granule cells layer of olfactory). 


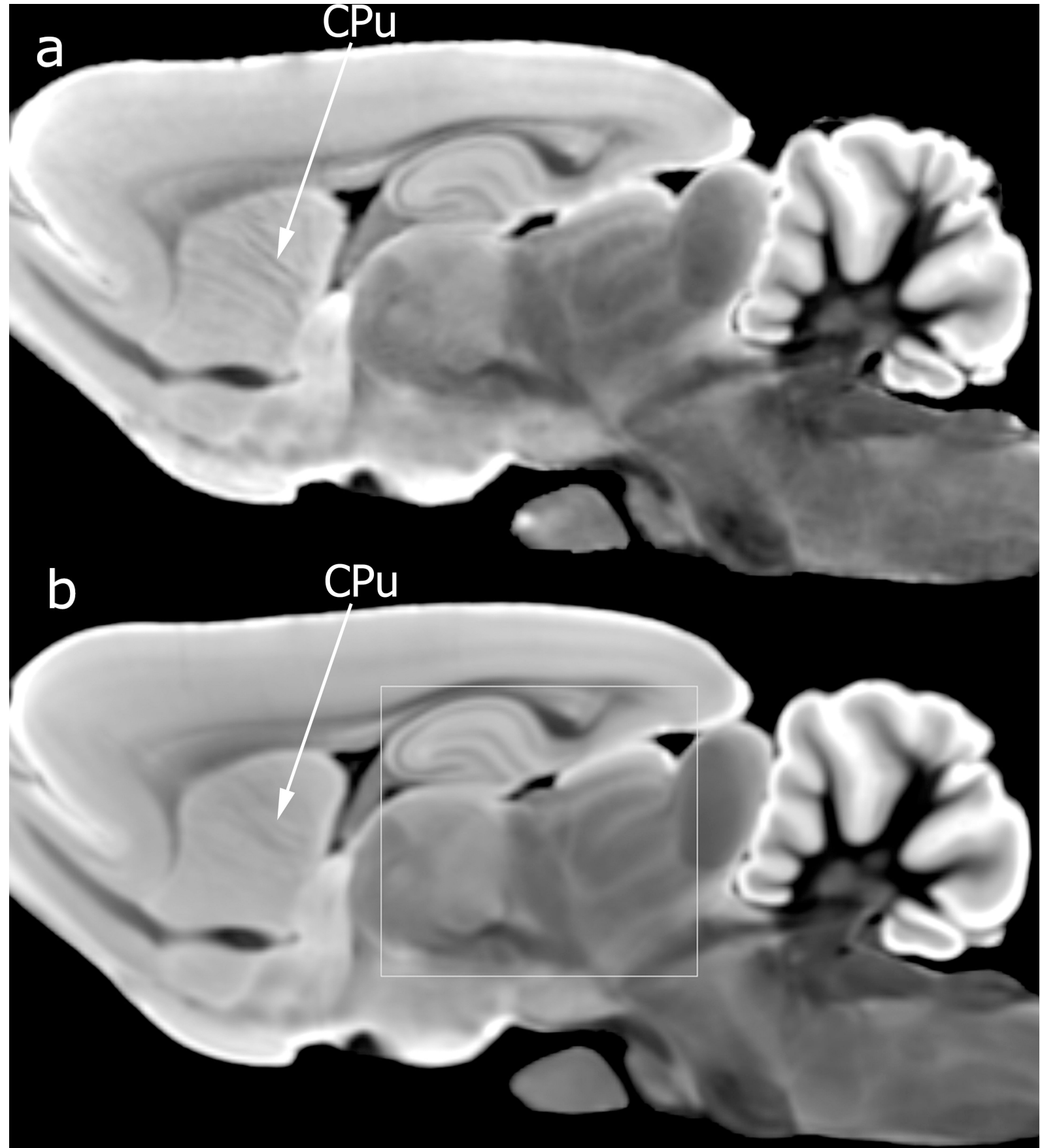

Fig. 4.

Sagittal slice through a control rat brain showing (a) diffusion weighted image (DWI), and (b) the minimum deformation template obtained from the 8 diffusion weighted images from the control specimens (inset shows DWI through the hippocampus, magnified in Fig. 5b). Notice white matter striations (arrows) within the caudate putamen $(\mathrm{CPu})$ in the single specimen (a), relative to the minimum deformation template (b). 


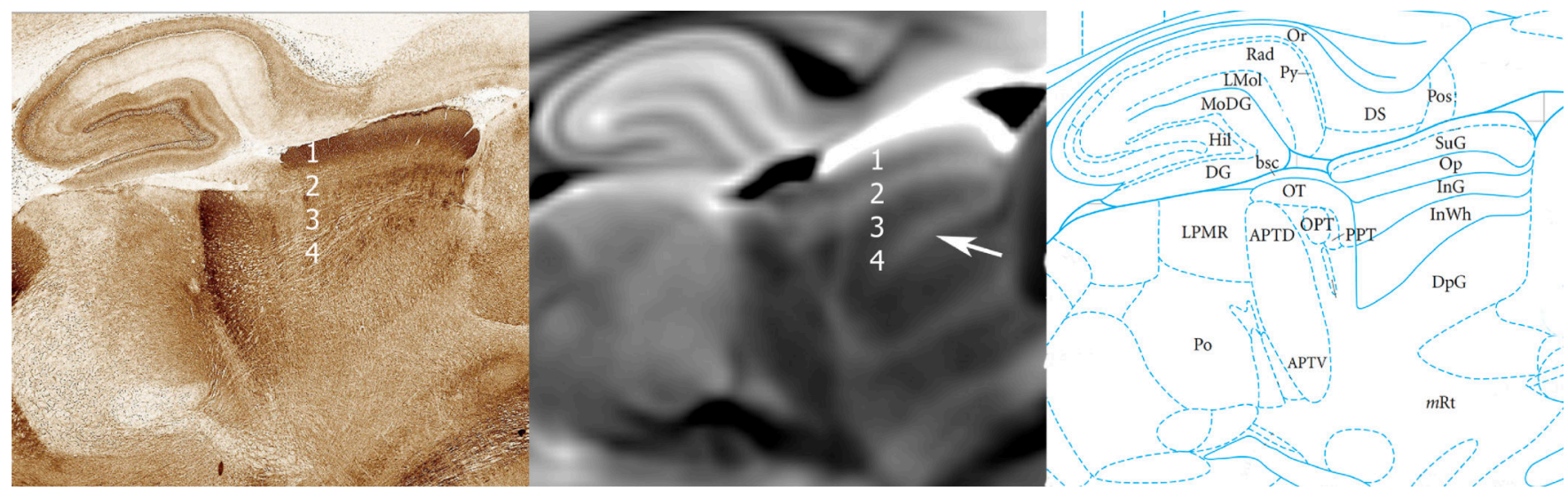

Fig. 5.

Comparison of (a) conventional AChE histology from Paxinos and Watson atlas (2007) and (b) diffusion weighted image (DWI) through the hippocampus (see inset in Fig. 4b), with (c) labels of cellular layers in the Paxinos and Watson atlas demonstrate how well MR delineates the boundaries in many of these subtle neuroanatomical structures (arrow in Fig. 5b) (adapted from and reprinted with permission from Elsevier Academic Press, San Diego, Plate 169 in: George Paxinos and Charles Watson, The Rat Brain in Stereotaxic Coordinates $6^{\text {th }} e d$., ISBN-13 978-0125476126, 2007). The superior colliculus layers depicted include: (1) SuG (superficial gray); (2) Op (optic nerve layer); (3) InG (internal gray layer); (4) InWh (internal white layer); as well as immediately ventral the DpG (deep gray layer). The hippocampal layers are also clearly visible: Or (oriens); Rad (stratum radiatum); LMol (lacunosum moleculare); MoDG (molecular layer of dentate gyrus); DG (dentate gyrus); Hil (hilus). 


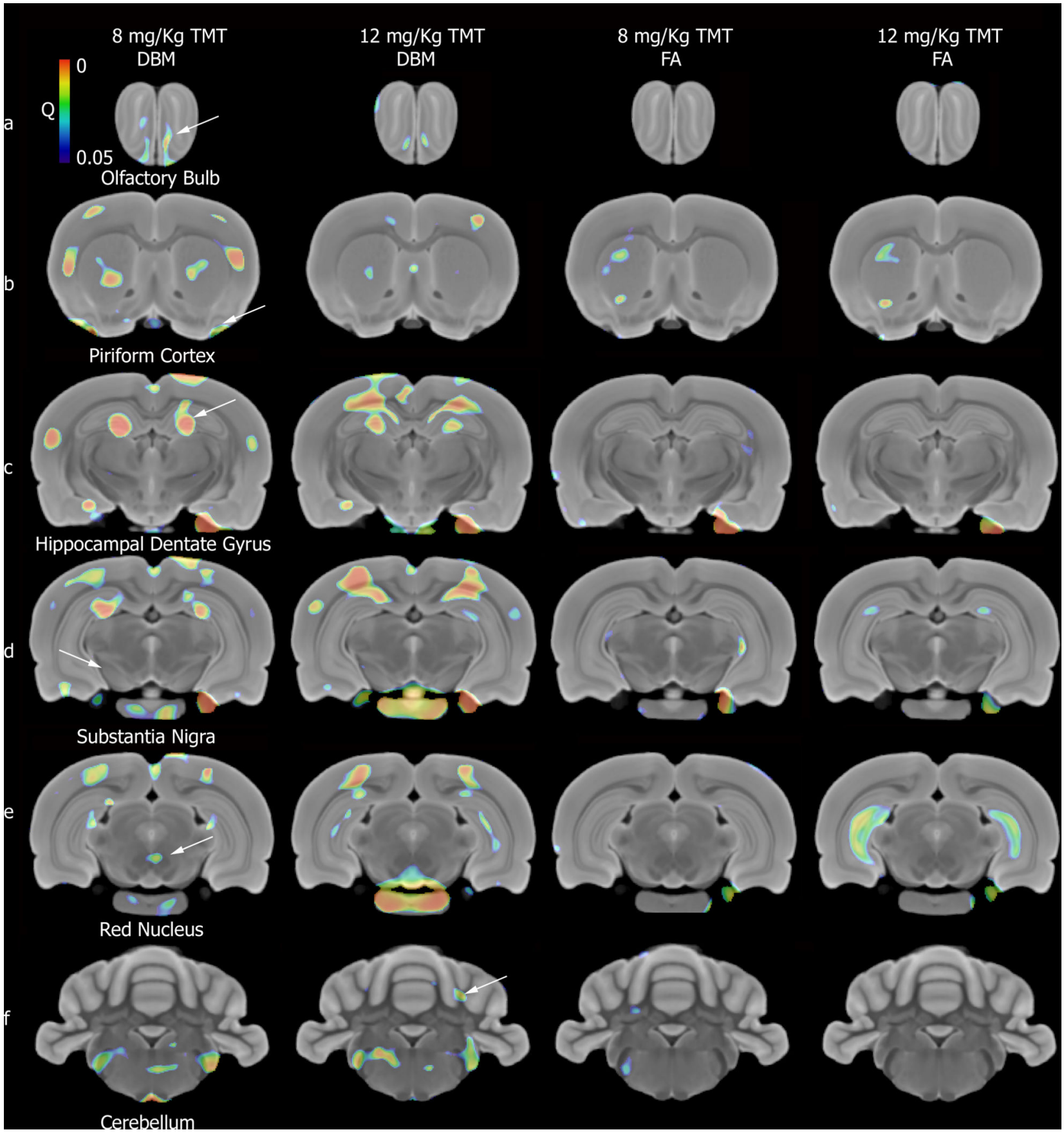

Fig. 6.

The corrected $\mathrm{p}$ values $(\mathrm{Q})$ are superimposed on the average diffusion weighted image from the control group demonstrating areas where there is morphologic difference between the control and $8 \mathrm{mg} / \mathrm{Kg}$-dose groups (column 1) and control and $12 \mathrm{mg} / \mathrm{Kg}$-dose group (column 2). A potential confound we need to acknowledge is due to the 3 days difference in the time point evaluation, where for humane reasons, rats on $12 \mathrm{mg} / \mathrm{Kg}$ were sacrificed earlier than rats in the $8 \mathrm{mg} / \mathrm{Kg}$-dose cohort. Regions in which there are differences in the FA are shown for comparison between the control and low-dose (column 3) and control and high-dose 
group (column 4). White arrows indicate anatomical regions identified by $\mathrm{H} \& \mathrm{E}$ as different in TMT-treated rats. 

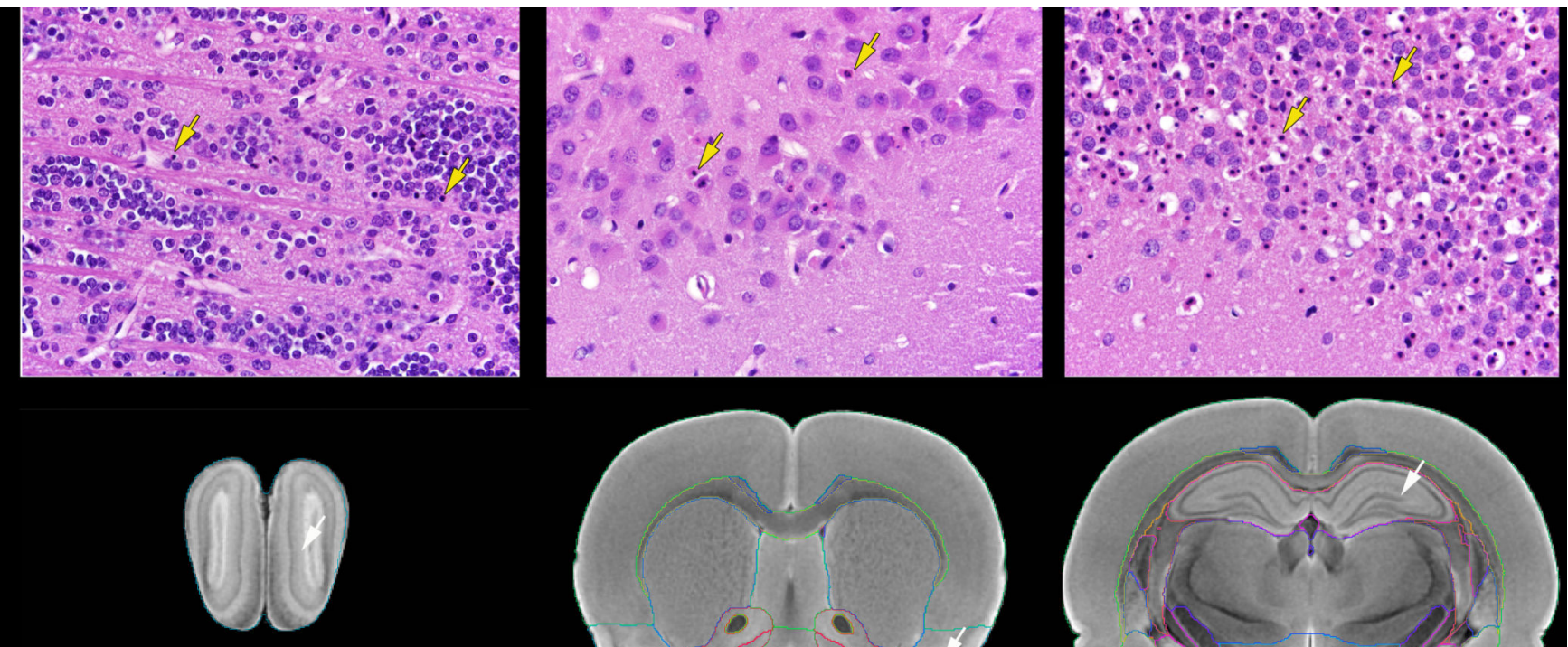

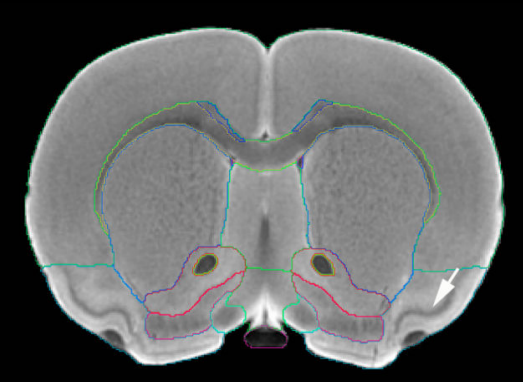

b) Piriform Cortex

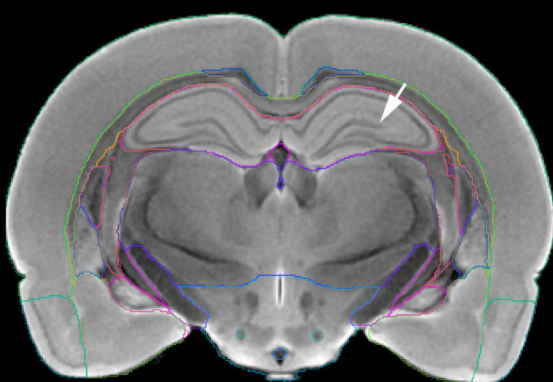

c) Hippocampal Dentate Gyrus

\section{a) Olfactory Bulb}
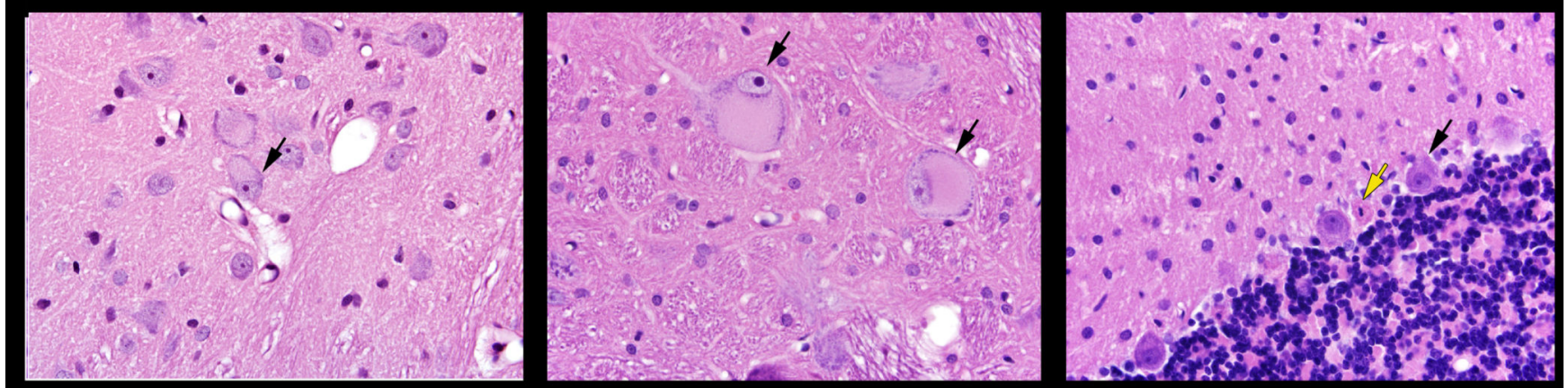

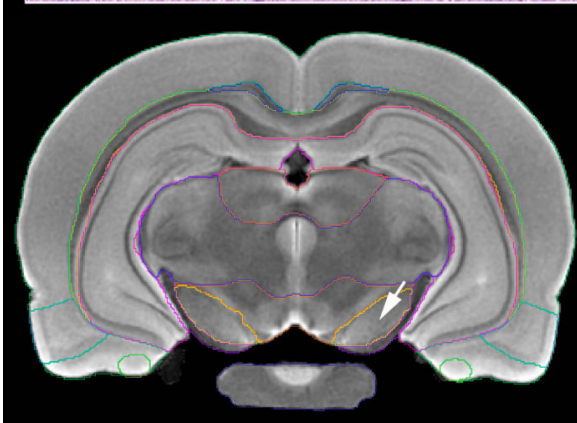

d) Substantia Nigra

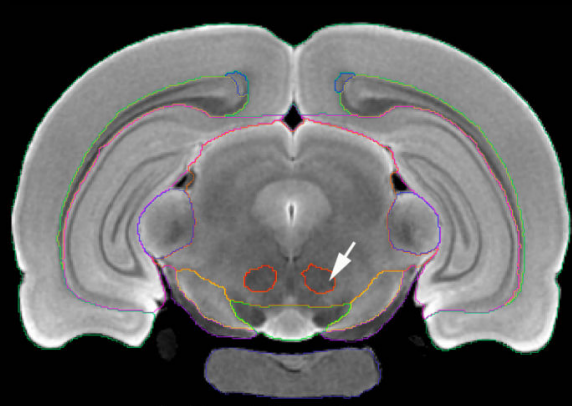

e) Red Nucleus

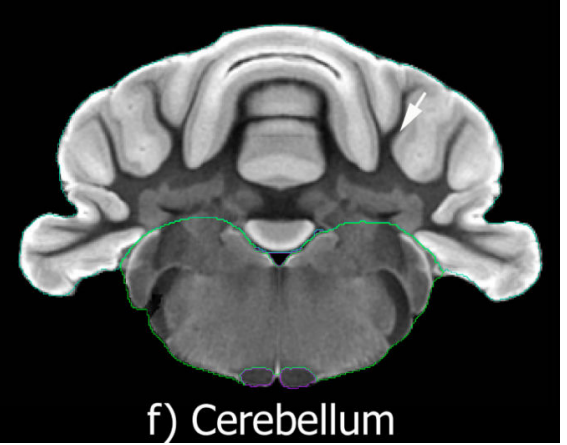

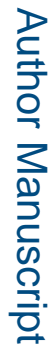

Fig. 7.

H\&E sections through: a) the olfactory bulb; b) piriform cortex; c) hippocampal dentate gyrus; d) substantia nigra; e) red nucleus, and f) cerebellum show widely dispersed evidence of necrosis (yellow arrows) and neuronal central chromatolysis (black arrows) in $12 \mathrm{mg} / \mathrm{Kg}$ TMT-treated rats. White arrows on MRH images indicate the approximate location for the $\mathrm{H} \& \mathrm{E}$ section details on the magnetic resonance histology reference atlas (Johnson et al. 2012). 


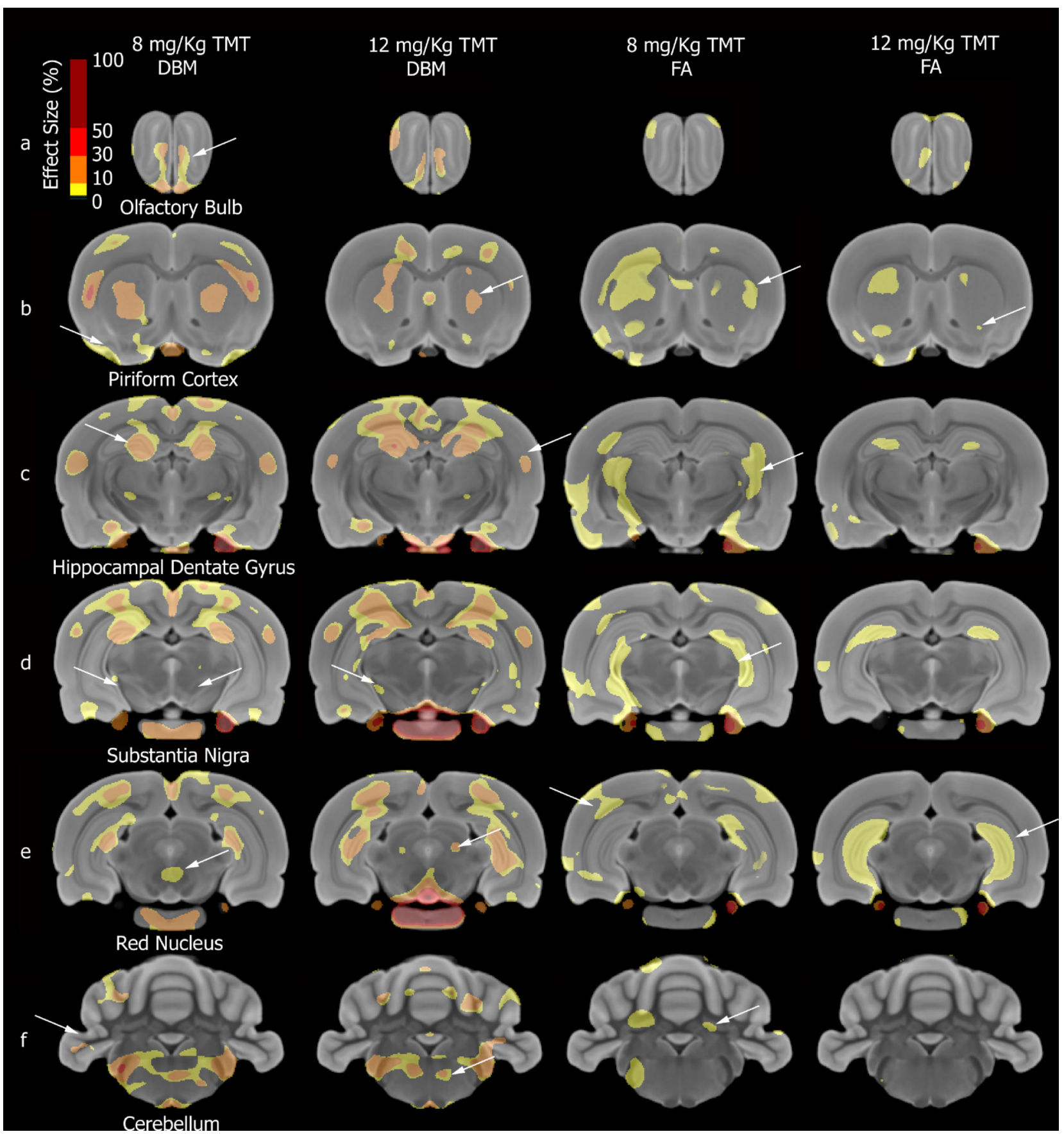

Fig. 8.

Statistical maps showing the effect size for local volume changes and FA parameters (FDR 0.15 ) between the two TMT-treated animal cohorts, relative to controls. Effect sizes are attributed to TMT dose, but we should acknowledge a difference of 3 days in time points for the $12 \mathrm{mg} / \mathrm{Kg}$-group. Effect sizes are assigned into one of four categories, from low $(<10 \%)$, to mild (10-30\%), medium (30-50\%), and large ( $>50 \%)$ effects. The same coronal sections as in Fig. 6 examined for local changes in DTI parametric maps after correcting for morphometric changes. Regions with significant differences in fractional anisotropy (FA) 
between TMT-treated rats and saline injected controls include: (1) granule cells layer in the olfactory bulbs; (2) the piriform cortex and striatum, as well as external capsule; (3) dentate gyrus of hippocampus (well defined in the $12 \mathrm{mg} / \mathrm{Kg}$-dose treated group), cerebellar nuclei, and white matter. 


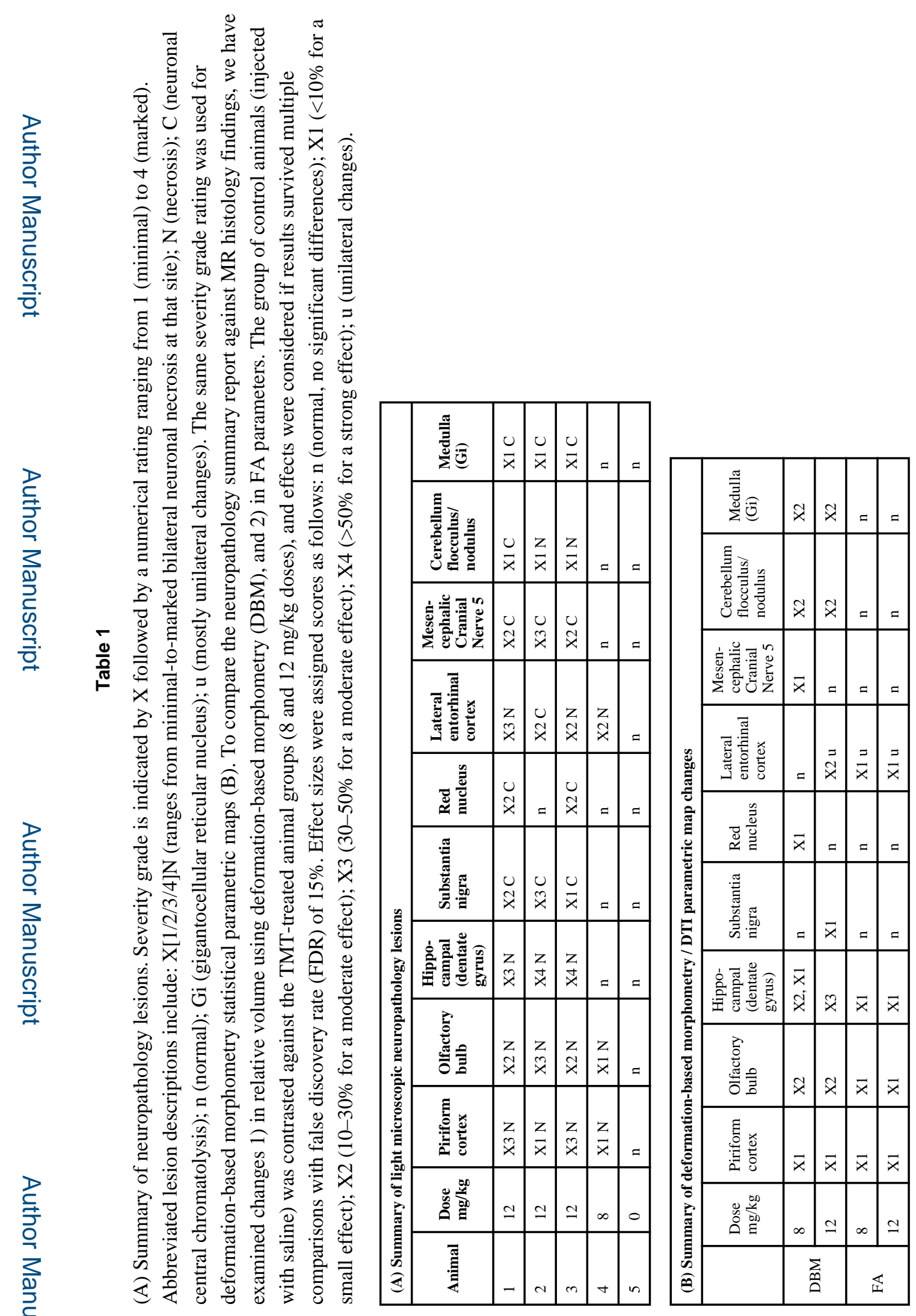

